Article

\title{
Associations between Unhealthy Diet and Lifestyle Behaviours and Increased Cardiovascular Disease Risk in Young Overweight and Obese Women
}

\author{
Megan C. Whatnall ${ }^{1}$, Clare E. Collins ${ }^{1}$, Robin Callister ${ }^{2}$ and Melinda J. Hutchesson ${ }^{1, *}$ \\ 1 School of Health Sciences, Faculty of Health and Medicine, and Priority Research Centre in Physical Activity \\ and Nutrition, University of Newcastle, Callaghan 2308, Australia; megan.whatnall@uon.edu.au (M.C.W.); \\ clare.collins@newcastle.edu.au (C.E.C.) \\ 2 School of Biomedical Sciences and Pharmacy, Faculty of Health and Medicine, and Priority Research Centre \\ in Physical Activity and Nutrition, University of Newcastle, Callaghan 2308, Australia; \\ robin.callister@newcastle.edu.au \\ * Correspondence: Melinda.Hutchesson@newcastle.edu.au; Tel.: +61-2-49-215405
}

Academic Editor: Sampath Parthasarathy

Received: 17 June 2016; Accepted: 12 August 2016; Published: 19 August 2016

\begin{abstract}
Unhealthy lifestyle behaviours are known modifiable risk factors for cardiovascular disease (CVD). This cross-sectional analysis aimed to describe lifestyle behaviours and CVD risk markers in young overweight and obese Australian women and explore associations between individual and combined lifestyle behaviours with CVD risk markers. Lifestyle behaviours assessed were diet quality, alcohol intake, physical activity, sitting time and smoking status, and were combined to generate a Healthy Lifestyle Score (HLS) (0-5). Objectively measured CVD risk markers were body mass index (BMI), \%body fat, waist circumference, blood pressure, and plasma cholesterol and triglycerides. Analysis included 49 women aged 18-35 years, with BMI 25.0 to $34.9 \mathrm{~kg} / \mathrm{m}^{2}$. The mean $\pm \mathrm{SD}$ Australian Recommended Food Score was $33.5 \pm 9.3$ points, alcohol $3.3 \pm 2.4$ standard drinks/day, physical activity $207 \pm 225 \mathrm{~min} /$ week and sitting time $578 \pm 213 \mathrm{~min} /$ day. All participants were non-smokers. The proportion of participants outside normal reference ranges was $83.7 \%$ for waist circumference $(n=41)$, blood pressure $0 \%(n=0)$, total cholesterol $26.2 \%(n=11)$, HDL cholesterol 38.6\% $(n=17)$, LDL cholesterol $22.7 \%(n=10)$, and triglycerides $4.2 \%(n=2)$. Physical activity was inversely associated with body fat $(\beta=-0.011 \%, p=0.005)$, diastolic blood pressure $(\beta=-0.010 \mathrm{mmHg}, p=0.031)$ and waist circumference $(\beta=-0.013 \mathrm{~cm}, p=0.029)$. Most participants $(59.2 \%, n=29)$ had a HLS $\leq 2$. No significant associations were found between HLS and CVD risk markers. Insufficient physical activity was the primary lifestyle factor associated with increased CVD risk markers, which suggests interventions targeting physical activity in young women may potentially improve cardiovascular health.
\end{abstract}

Keywords: cardiovascular disease risk; nutrition; lifestyle behaviours; young women

\section{Introduction}

Cardiovascular disease (CVD) is the leading cause of total and premature death in women globally [1,2]. Lifestyle behaviours known to increase CVD risk include high alcohol consumption, poor diet quality, physical inactivity, extended sitting time and smoking [3-6]. The prevalence of these lifestyle behaviours in women is high, with $>90 \%$ of women in Australia and the USA reporting one or more risk behaviour [6,7]. In 2008, 34\% of adult women worldwide were not sufficiently physically active [8], while 53\% of 18-34-year-old Australian women are not meeting physical activity recommendations [9]. Most consume inadequate servings of fruits and vegetables, including $96 \%$ of 18-34-year-old Australian females [10]. A significant proportion of adult females smoke, at $7 \%$ 
globally [11], 15\% in America [12] and 16\% in Australia [13]. In the USA, of the $60 \%$ of adult women who drink alcohol, $13 \%$ consume more than the recommended daily amount [14]. In Australia 10\% of adult females exceed national alcohol guidelines of $\leq 2$ drinks/day [15]. Studies of sitting time in US and Australian adult women indicate $51 \%$ and $75 \%$ respectively of participants spend $>3 \mathrm{~h}$ sitting/day $[5,16]$.

Several observational studies have demonstrated that higher CVD risk is conferred by a greater number of risky lifestyle behaviours [17-22]. For example, a prospective cohort study of Swedish women aged 49-83 years investigated the association between a low risk lifestyle pattern (healthy diet, moderate alcohol consumption, never smoking, being physically active, healthy body mass index (BMI)) and stroke risk. Over the ten-year follow-up, those with all five low risk lifestyle behaviours had a 54\% lower risk of stroke than those with none [17]. This relationship is supported by similar studies in middle and older-aged males and females [18-20]. Yang et al. examined the association between seven cardiovascular health metrics (not smoking, physically active, normal blood pressure, blood glucose and total cholesterol levels, normal weight and a healthy diet) and CVD mortality in a US adult cohort [20]. Over a median follow-up of 14.5 years, adults with six or more healthy behaviours had an absolute CVD mortality risk of $15 \%$ compared to $65 \%$ for those reporting only one or no healthy behaviours [20].

O'Flaherty et al. in a time trend analysis of Australian men and women found that the current 30-year trend of declining coronary heart disease (CHD) mortality is slowing, suggesting that young adults will experience greater CHD morbidity and mortality compared to the current generation of older adults [23]. The high prevalence of risk behaviours in young adults currently supports this trend [6-8,10-16,24]. However, few studies have specifically investigated young adults' lifestyle behaviours and CVD risk. The Coronary Artery Risk Development in Young Adults (CARDIA) study examined whether a healthy lifestyle (never smoking, healthy BMI, no/moderate alcohol intake and higher healthy diet and physical activity scores) in young adulthood (18-30 years) was associated with a low CVD risk profile (no pre-existing CVD, untreated cholesterol $<200 \mathrm{mg} / \mathrm{dL}$, untreated blood pressure $<120 /<80 \mathrm{mmHg}$, never smoking, and no history of diabetes or myocardial infarction) in middle age [21]. The prevalence of a low CVD risk profile was $60 \%$ for those with five healthy lifestyle factors compared to $3 \%$ for those with one [21]. Chomistek et al. conducted a similar study in young and middle-aged women (27-44 years at baseline) exploring the association between a healthy lifestyle (no smoking, healthy BMI, higher diet quality, no/moderate alcohol intake, no/moderate television viewing and being physically active) and clinical CVD risk factors (diabetes, hypertension and hypercholesterolaemia) over a 20-year follow-up [22]. They found that $46 \%$ of clinical CVD risk factor cases were attributable to a poor lifestyle [22].

One study has specifically explored the direct association between lifestyle behaviours during young adulthood and CVD risk [25]. Gall et al. examined cross-sectionally the association between a healthy lifestyle (healthy BMI, non-smoker, low alcohol, physically active and healthy dietary behaviours) and CVD risk factors (blood pressure, LDL and HDL cholesterol, triglycerides, glucose, insulin and insulin resistance) in young Australian adults (25-36 years) [25]. Significant associations were found between a healthy lifestyle and HDL cholesterol $(\beta=0.02)$ and glucose $(\beta=-0.02)$ in females, with a number of significant associations also found for the male study participants [25]. These findings suggest that unhealthy lifestyle behaviours in young adulthood are having a direct impact on cardiovascular health, and that further exploration of such an association in this age group is needed.

The current study investigated these relationships at baseline in young overweight and obese women (18-35 years) enrolled in a weight-loss study. The first aim was to describe the young women's lifestyle behaviours (diet quality, alcohol intake, physical activity, sitting time and smoking) and CVD risk markers (BMI, \%body fat, waist circumference, blood pressure, total, HDL and LDL cholesterol and triglycerides). The second aim was to examine associations between lifestyle behaviours and CVD risk markers. The final aim was to explore associations between a total Healthy Lifestyle Score (HLS) 
that summarises overall diet quality, alcohol intake, physical activity, sitting time and smoking status, and CVD risk markers. It was hypothesised that the study population would have unhealthy lifestyle behaviours and elevated CVD risk markers, and that unhealthy lifestyle behaviours and a lower HLS would be associated with higher CVD risk.

\section{Materials and Methods}

\subsection{Study Design}

This study was a cross-sectional analysis of baseline data from the Be Positive Be Healthe randomised controlled trial (RCT), which evaluated efficacy of a weight-loss program targeting young (18-35 years) overweight and obese women.

\subsection{Participants and Recruitment}

RCT inclusion criteria were: female, $18-35$ years, BMI $25.0-34.9 \mathrm{~kg} / \mathrm{m}^{2}$, email and internet access, iPhone model 4 s or newer, social media accounts willing to be used for the study and able to attend measurement sessions. Participants were excluded if: currently pregnant or breastfeeding, planning pregnancy in next 6 months, participating in another weight-loss program, taking medications which had caused weight gain, had a metabolic disorder, eating disorder or other medical condition where weight loss may compromise health, non-English speaking or had weight loss of $\geq 5 \%$ of initial weight in the last three months.

Participants were recruited via media releases by the University of Newcastle and Hunter Medical Research Institute, posters at the university campus, local technical college, local businesses and organisations known to engage with the target group, and social media pages of these settings [26]. Email invitation was also sent to consenting participants of a previous research study.

All participants gave written informed consent prior to participating in the study. The study was conducted in accordance with the Declaration of Helsinki and the protocol was approved by the University of Newcastle Human Research Ethics Committee (H-2014-0138).

\subsection{Measures}

An online questionnaire collected self-reported data on dietary and alcohol intakes, physical activity, sitting time and smoking status using Survey Monkey (www.surveymonkey.com.au). Participants attended the University of Newcastle campus for objective measurement of CVD risk markers. The online questionnaires were completed within the two days prior to or during the baseline measurement session.

\subsubsection{Exposure: Measurement of Lifestyle Behaviours}

Dietary intake was assessed using the validated Australian Eating Survey food frequency questionnaire (AES FFQ) [27], a self-administered 120-item semi-quantitative FFQ which asks respondents to report usual intake over the previous six months. Diet quality was determined using the validated Australian Recommended Food Score (ARFS) [28], calculated using a sub-set of 70 items from the AES FFQ relating to intake of vegetables, fruit, meat/flesh foods, non-meat/flesh protein foods, breads and cereals, dairy foods, water and spreads/sauces. The ARFS adds the points for each item, with most foods attributed one point for a consumption frequency of $\geq$ once per week, and the total score ranging from zero to 73 points. A higher score is indicative of greater dietary variety, more optimal nutrient intakes [28] and dietary patterns more aligned with the Australian Dietary Guidelines [29].

Alcohol intake was assessed using questions from the New South Wales Adult Population Health Survey [30], based on participants' alcohol consumption over the previous six months. Participants were asked how many standard drinks they usually consumed on an occasion where they drank alcohol. 
Physical activity was assessed using the Godin Leisure Time Exercise Questionnaire [31]. Participants indicated the number of sessions per week and time (minutes) per session spent performing vigorous, moderate and mild intensity physical activity, based on the previous month. Total physical activity (min/week) was calculated as the sum of the number of sessions per week $\times$ time per session for vigorous and moderate activity.

Sitting time was assessed using the validated Domain-Specific Sitting Time Questionnaire [32]. Participants were asked to indicate the time (minutes) spent sitting/day in the previous month in five different situations (during travel, at work, watching television, using a computer at home, and in leisure time excluding television viewing), on a weekday and on a weekend day. Total sitting time/day for weekdays and weekend days was determined as the sum of sitting time for each situation. The average sitting time/day was calculated ((total time spent sitting on a weekday $\times 5)+($ total time spent sitting on a weekend day $\times 2)) / 7$.

Participants were asked if they currently smoke tobacco products, and if so if they have smoked at least 100 cigarettes or a similar amount of tobacco in their life. Participants were classed as a "smoker" if they reported smoking "daily" or smoking "at least once a week" or "less often than once a week" and smoking 100 cigarettes or a similar amount in their life. Participants were otherwise classed as a "non-smoker".

All five lifestyle behaviours (diet quality, alcohol intake, physical activity, sitting time and smoking) were included in defining the HLS. Similar to a number of previous studies [17-22] participants were awarded one point for each healthy lifestyle behaviour, therefore the HLS ranged from zero to five points, with a higher score indicating a healthier lifestyle. Points were attributed based on meeting population-based guidelines if available [33,34]. If no population-based recommendations were available, cut-points were based on the participants' data, with the highest/lowest quintile used. Therefore, participants were awarded one point for: an ARFS in the top quintile of the distribution of the cohort, alcohol intake of $\leq 2$ standard drinks on a day when alcohol is consumed [33], an accumulated $150 \mathrm{~min}$ of moderate, $75 \mathrm{~min}$ of vigorous or equivalent combination of moderate and vigorous physical activity/week [35], average sitting time in the lowest quintile of the distribution of the cohort, and if they were a non-smoker.

\subsubsection{Outcomes: Cardiovascular Disease (CVD) Risk Markers}

Height was measured to $0.1 \mathrm{~cm}$ using the stretch stature method on a stadiometer (Inbody BSM370; Inbody Australia, Miami, QLD, Australia). Weight to $0.01 \mathrm{~kg}$ was measured in light clothing, without shoes on a digital scale and body fat percentage determined using bioelectrical impedance (Inbody 720; Inbody Australia, Miami, QLD, Australia). BMI was calculated using the standard equation (weight $(\mathrm{kg}) /$ height $\left(\mathrm{m}^{2}\right)$ ) [36]. Waist circumference was measured to $0.1 \mathrm{~cm}$ using a non-extensible steel tape measure as the narrowest point between the lower costal border and the umbilicus. Blood pressure was measured using the automatic sphygmomanometer (Inbody BPBIO320, Inbody Australia, Miami, QLD, Australia). Participants were seated for five minutes prior to the first blood pressure measurement, with two minutes rest between additional measures. Total, HDL and LDL cholesterol and triglycerides were measured in a fasting blood sample (minimum $8 \mathrm{~h}$, maximum $12 \mathrm{~h}$ ). Blood samples were obtained by finger prick and analysed using the validated Cardiochek reflectance spectrophotometer lipid measurement tool (Point of Care Diagnostics Pty Ltd, Artarmon, NSW, Australia) [37,38]. All measurements for height, weight, waist circumference and blood pressure were taken twice for accuracy, with a third measurement also taken in cases where either of the first two values fell outside a predetermined acceptable range.

\subsubsection{Socio-Demographic Characteristics}

Socio-demographic data were collected including age, education, qualifications, income, ethnicity and postcode. Postcode of residence was used as an indicator of socio-economic status based on the Australian Bureau of Statistics Index of Relative Socio-Economic Advantage and Disadvantage (IRSAD) [39]. 


\subsection{Statistical Analysis}

Data analysis was conducted using Stata statistical software version 14.1 (StataCorp, College Station, TX, USA). Descriptive statistics were used to describe lifestyle behaviours, CVD risk markers and socio-demographic variables including standard deviations (SD) and means for continuous variables, and percentages for categorical variables. Unadjusted linear regression models were used to explore the association between individual lifestyle behaviours (diet quality, alcohol intake, physical activity and sitting time) and each CVD risk marker (BMI, \%body fat, waist circumference, blood pressure, total, HDL and LDL cholesterol and triglycerides). Each linear regression model was then repeated (adjusted model), to also include the other lifestyle behaviours, due to potential confounding. Finally, linear regression was used to explore the association between HLS and each CVD risk marker. All linear regression models were controlled for age, ethnicity and socio-economic status. Statistical significance was set at $p<0.05$.

\section{Results}

\subsection{Socio-Demographic Status}

A total of 57 women were recruited, with 49 included in the analyses. The remaining were excluded due to missing physical activity data $(n=5)$ or implausible sitting time data $(n=3)$. Participants' sociodemographic characteristics are summarised in Table 1. The mean \pm SD age of participants was $27.1 \pm 4.5$ years. The majority was currently studying $(55.1 \%)$, and were university students $(53.1 \%)$. The greatest proportion of participants had completed a university or higher university degree $(42.9 \%)$, followed by a certificate/diploma $(26.5 \%)$. The majority were born in Australia (93.9\%), including 2.0\% of Aboriginal descent. Most (42.9\%) reported a middle-range income (\$300-\$999/week), with a similar proportion (44.9\%) rating in the middle range (4-6) on the IRSAD.

\subsection{Lifestyle Behaviours}

Table 1 also summarises data for the five lifestyle behaviours. The mean \pm SD ARFS was $33.5 \pm 9.3$ points. The cut-point for the top quintile was $\geq 40$ points, with $75.5 \%$ scoring $\leq 40$ points. The mean number of standard drinks consumed/day, on days when alcohol was consumed, was $3.3 \pm 2.4$ standard drinks, with $44.9 \%$ exceeding recommendations of $\leq 2$ standard drinks on any day when alcohol was consumed. The mean total physical activity (minutes/week) was $207 \pm 225$, with almost half $(49.0 \%)$ reporting insufficient physical activity. The mean time spent sitting was $499 \pm 200 \mathrm{~min}$ on a weekend day and $610 \pm 260 \mathrm{~min}$ on a weekday, with an average sitting time of $578 \pm 213 \mathrm{~min} /$ day. The cut-point for the lowest quintile of sitting time was $\leq 394 \mathrm{~min} /$ day. Based on the average sitting time, $81.6 \%$ of participants sat for $\geq 394 \mathrm{~min} /$ day. All participants were non-smokers.

\subsection{CVD Risk Markers}

Table 1 summarises data on CVD risk markers. The mean BMI was $29.4 \pm 2.6 \mathrm{~kg} / \mathrm{m}^{2}$, with $44.9 \%$ of participants obese. Mean \%body fat was $37.7 \% \pm 5.7 \%$. Mean waist circumference was $88.8 \pm 8.6 \mathrm{~cm}$, with most participants $(83.7 \%)$ being rated at increased risk of metabolic complications $(\geq 80 \mathrm{~cm})$, and close to half $(49.0 \%)$ at substantially increased risk of metabolic complications $(\geq 88 \mathrm{~cm})$ [40]. The mean systolic blood pressure was $112 \pm 9 \mathrm{mmHg}$, and $74 \pm 8 \mathrm{mmHg}$ for diastolic. No participants had hypertension $(>140 / 90 \mathrm{mmHg}$ ), however $10.0 \%$ had blood pressure in the normal-high range $(>120 / 80 \mathrm{mmHg})$ [41]. Mean total cholesterol was $5.0 \pm 0.9 \mathrm{mmol} / \mathrm{L}$, with $33.3 \mathrm{~s} \%$ of plasma total cholesterol concentrations $\geq 5.5 \mathrm{mmol} / \mathrm{L}$ [42]. Mean HDL cholesterol was $1.5 \pm 0.4 \mathrm{mmol} / \mathrm{L}$, with $38.6 \%$ of plasma HDL cholesterol concentrations $<1.3 \mathrm{mmol} / \mathrm{L}$ [42]. Mean LDL cholesterol was $2.9 \pm 0.8 \mathrm{mmol} / \mathrm{L}$, with $22.7 \%$ of participants with concentrations $\geq 3.5 \mathrm{mmol} / \mathrm{L}$ [42]. The mean plasma triglyceride concentration was $1.2 \pm 0.5 \mathrm{mmol} / \mathrm{L}$, with $4.2 \%$ of participants triglycerides $\geq 2.0 \mathrm{mmol} / \mathrm{L}$ [43]. 
Table 1. Baseline characteristics of young (18-35 years) overweight and obese women (BMI $\left.25-34.9 \mathrm{~kg} / \mathrm{m}^{2}\right)$ entering a weight-loss trial $\left(n=49{ }^{1}\right)$, by Healthy Lifestyle Score.

\begin{tabular}{|c|c|c|c|c|c|c|c|c|c|c|c|c|c|}
\hline \multirow{3}{*}{ Variable } & \multicolumn{12}{|c|}{ Healthy Lifestyle Score } & \multirow{3}{*}{$p$} \\
\hline & \multicolumn{2}{|c|}{ All participants } & \multicolumn{2}{|l|}{1} & \multicolumn{2}{|l|}{2} & \multicolumn{2}{|l|}{3} & \multicolumn{2}{|l|}{4} & \multicolumn{2}{|l|}{5} & \\
\hline & Mean or $\mathrm{n}(\%)$ & SD & Mean or $n(\%)$ & SD & Mean or $n(\%)$ & SD & Mean or $\mathbf{n}(\%)$ & SD & Mean or $n(\%)$ & SD & Mean or $n(\%)$ & SD & \\
\hline $\mathrm{N}$ & $49(100.0)$ & - & $5(10.2)$ & 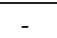 & $24(49.0)$ & 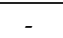 & $12(24.5)$ & 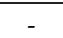 & $7(14.3)$ & 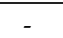 & $1(2.0)$ & - & 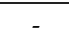 \\
\hline Age (years) & 27.1 & 4.5 & 27 & 5.2 & 27.4 & 4.3 & 27.8 & 4.7 & 25.5 & 5.2 & 22.1 & - & 0.56 \\
\hline Completed year 12 or equivalent & $46(93.9)$ & - & $5(100.0)$ & - & $23(95.8)$ & - & $10(83.3)$ & - & $7(100.0)$ & - & $1(100.0)$ & - & - \\
\hline $\begin{array}{l}\text { Currently studying } \\
\text { Highest qualification }\end{array}$ & $27(55.1)$ & - & $3(60.0)$ & - & $13(54.2)$ & - & $8(66.7)$ & - & $2(28.6)$ & - & $1(100.0)$ & - & $\begin{array}{c}0.5 \\
0.35\end{array}$ \\
\hline Trade/Apprenticeship & $2(4.1)$ & - & $0(0.0)$ & - & $1(4.2)$ & - & $1(8.3)$ & - & $0(0.0)$ & - & $0(0.0)$ & - & - \\
\hline Certificate/Diploma & $13(26.5)$ & - & $0(0.0)$ & - & $9(37.5)$ & - & $3(25.0)$ & - & $1(14.3)$ & - & $0(0.0)$ & - & - \\
\hline $\begin{array}{l}\text { University/Higher University } \\
\text { Degree }\end{array}$ & $21(42.9)$ & - & $4(80.0)$ & - & $8(33.3)$ & - & $4(33.3)$ & - & $5(71.4)$ & - & $0(0.0)$ & - & - \\
\hline Ethnicity & & & & & & & & & & & & & 0.96 \\
\hline Australian & $46(93.9)$ & - & $4(80.0)$ & - & $23(95.8)$ & - & $12(100.0)$ & - & $6(85.7)$ & - & $1(100.0)$ & - & - \\
\hline Other & $3(6.1)$ & - & $1(20.0)$ & - & $1(4.2)$ & - & $0(0.0)$ & - & $1(14.3)$ & - & $0(0.0)$ & - & - \\
\hline Aboriginal origin & $1(2.0)$ & - & $0(0.0)$ & - & $0(0.0)$ & - & $1(8.3)$ & - & $0(0.0)$ & - & $0(0.0)$ & - & 1.00 \\
\hline Weekly income & & & & & & & & & & & & & 0.23 \\
\hline Lower (\$0-\$299) & $12(24.5)$ & - & $1(20.0)$ & - & $2(8.3)$ & - & $6(50.0)$ & - & $2(28.6)$ & - & $1(100.0)$ & - & - \\
\hline Middle (\$300-\$999) & $21(42.9)$ & - & $3(60.0)$ & - & $12(50.0)$ & - & $3(25.0)$ & - & $3(42.9)$ & - & $0(0.0)$ & - & - \\
\hline Higher $(\geq \$ 1000)$ & $16(32.6)$ & - & $1(20.0)$ & - & $10(41.7)$ & - & $3(25.0)$ & - & $2(28.6)$ & - & $1(0.0)$ & - & - \\
\hline SES (IRSAD) & & & & & & & & & & & & & 0.64 \\
\hline $1-3$ & $11(22.5)$ & - & $1(20.0)$ & - & $6(25.0)$ & - & $3(25.0)$ & - & $1(14.3)$ & - & $0(0.0)$ & - & - \\
\hline $4-6$ & $22(44.9)$ & - & $3(60.0)$ & - & $10(41.7)$ & - & $6(50.0)$ & - & $2(28.6)$ & - & $1(100.0)$ & - & - \\
\hline $7-9$ & $16(32.6)$ & - & $1(20.0)$ & - & 8 (33.5) & - & $3(25.0)$ & - & $4(57.2)$ & - & $0(0.0)$ & - & - \\
\hline ARFS & 33.5 & 9.3 & 24.6 & 5.3 & 31.8 & 9.1 & 35.9 & 8.7 & 40.6 & 7.2 & 42.0 & - & 0.01 \\
\hline Top quintile ( $\geq 40$ ) & $12(24.5)$ & - & $0(0.0)$ & - & $2(8.3)$ & - & $4(33.3)$ & - & 5 (71.4) & - & $1(100.0)$ & - & - \\
\hline $\begin{array}{l}\text { Alcohol intake (standard } \\
\text { drinks/day) }\end{array}$ & 3.3 & 2.4 & 4.0 & 1.0 & 3.3 & 2.6 & 3.1 & 2.2 & 3.1 & 3.1 & 2.0 & - & 0.63 \\
\hline Meeting HLC & $27(55.1)$ & - & $0(0.0)$ & - & $14(58.3)$ & & $7(58.3)$ & & $5(71.4)$ & & $1(100.0)$ & - & \\
\hline Total physical activity (min/week) & 207 & 225 & 42 & 61 & 145 & 155 & 282 & 188 & 399 & 391 & 300 & - & 0.003 \\
\hline Meeting HLC & $25(51.0)$ & - & $0(0.0)$ & - & $8(33.3)$ & - & $9(75.0)$ & - & $7(100.0)$ & - & $1(100.0)$ & - & - \\
\hline
\end{tabular}


Table 1. Cont.

\begin{tabular}{|c|c|c|c|c|c|c|c|c|c|c|c|c|c|}
\hline \multirow{3}{*}{ Variable } & \multicolumn{12}{|c|}{ Healthy Lifestyle Score } & \multirow{3}{*}{$p$} \\
\hline & \multicolumn{2}{|c|}{ All participants } & \multicolumn{2}{|l|}{1} & \multicolumn{2}{|l|}{2} & \multicolumn{2}{|l|}{3} & \multicolumn{2}{|l|}{4} & \multicolumn{2}{|l|}{5} & \\
\hline & Mean or $\mathbf{n}(\%)$ & SD & Mean or $n(\%)$ & SD & Mean or $n(\%)$ & SD & Mean or $n(\%)$ & SD & Mean or $n(\%)$ & SD & Mean or $n(\%)$ & SD & \\
\hline Sitting time $(\min /$ day $)$ & & & & & & & & & & & & & \\
\hline Weekday & 610 & 260 & 530 & 158 & 722 & 274 & 538 & 234 & 433 & 175 & 450 & - & 0.03 \\
\hline Weekend day & 499 & 200 & 548 & 119 & 548 & 240 & 438 & 131 & 416 & 174 & 360 & - & 0.42 \\
\hline Average of days & 578 & 213 & 535 & 95 & 672 & 230 & 509 & 174 & 428 & 152 & 424 & - & 0.01 \\
\hline Lowest quintile ( $\leq 394)$ & $9(18.4)$ & - & $0(0.0)$ & - & $0(0.0)$ & - & $4(33.3)$ & - & $4(57.1)$ & - & $1(100.0)$ & - & 0.06 \\
\hline Non-smokers & 49 (100.0) & - & $5(100.0)$ & - & $24(100.0)$ & - & $12(100.0)$ & - & $7(100.0)$ & - & $1(100.0)$ & - & - \\
\hline BMI $\left(\mathrm{kg} / \mathrm{m}^{2}\right)$ & 29.4 & 2.6 & 29.9 & 2.9 & 29.2 & 2.7 & 28.4 & 2.0 & 31.8 & 1.5 & 28.9 & - & 0.08 \\
\hline Overweight & $27(55.1)$ & - & $2(40.0)$ & - & $14(58.3)$ & - & $9(75.0)$ & - & $1(14.3)$ & - & $1(100.0)$ & - & - \\
\hline Obese & $22(44.9)$ & - & $3(60.0)$ & - & $10(41.7)$ & - & $3(25.0)$ & - & $6(85.7)$ & - & $0(0.0)$ & - & - \\
\hline Body fat $\%$ & 37.7 & 5.7 & 39.4 & 7.6 & 37.5 & 5 & 35.7 & 6.5 & 40.2 & 4.9 & 40.3 & - & 0.57 \\
\hline Waist circumference $(\mathrm{cm})$ & 88.8 & 8.6 & 88.2 & 6.9 & 88.4 & 9.3 & 87.5 & 8.4 & 91.5 & 9.1 & 96.8 & - & 0.60 \\
\hline$\geq 80 \mathrm{~cm}$ & $17(34.7)$ & - & $3(60.0)$ & - & $10(41.7)$ & - & $3(25.0)$ & - & $1(14.3)$ & - & $0(0.0)$ & - & - \\
\hline$\geq 88 \mathrm{~cm}$ & $24(49.0)$ & - & $2(40.0)$ & - & $10(41.7)$ & - & $6(50.0)$ & - & $5(71.4)$ & - & $1(100.0)$ & - & - \\
\hline Systolic bloo $\bar{d}$ pressure $(\mathrm{mmHg})$ & 112.1 & 8.6 & 116.4 & 7.4 & 112.9 & 8.5 & 109.4 & 8.9 & 112 & 9.9 & 107 & - & 0.35 \\
\hline Diastolic blood pressure (mmHg) & 74.3 & 7.8 & 76.6 & 4.5 & 75.1 & 9.5 & 71.3 & 5.1 & 75.7 & 6.4 & 69 & - & 0.26 \\
\hline Total cholesterol $(\mathrm{mmol} / \mathrm{L})$ & 5.0 & 0.9 & 4.7 & 0.6 & 5.1 & 0.9 & 5.2 & 1.0 & 5.1 & 1.2 & 4.2 & - & 0.73 \\
\hline$\geq 5.5 \mathrm{mmol} / \mathrm{L}$ & $16(33.3)$ & - & $1(20.0)$ & - & $7(29.2)$ & - & $5(45.5)$ & - & $3(42.9)$ & - & $0(0.0)$ & - & - \\
\hline HD̄L-C $(\mathrm{mmol} / \mathrm{L})$ & 1.5 & 0.4 & 1.3 & 0.3 & 1.6 & 0.5 & 1.5 & 0.5 & 1.4 & 0.4 & 1.4 & - & 0.63 \\
\hline$<1.3 \mathrm{mmol} / \mathrm{L}$ & $17(38.6)$ & - & $4(80.0)$ & - & $7(33.3)$ & - & $3(30.0)$ & - & $3(42.9)$ & - & $0(0.0)$ & - & - \\
\hline LDL-C $(\mathrm{mmol} / \mathrm{L})$ & 2.9 & 0.8 & 2.9 & 0.7 & 2.8 & 0.7 & 3 & 0.7 & 3.1 & 1.0 & 2.3 & - & 0.89 \\
\hline$\geq 3.5 \mathrm{mmol} / \mathrm{L}$ & $10(22.7)$ & - & $1(20.0)$ & - & $4(19.1)$ & - & $2(20.0)$ & - & $3(42.9)$ & - & $0(0.0)$ & - & - \\
\hline Triglycerides $(\mathrm{mmol} / \mathrm{L})$ & 1.2 & 0.5 & 1.1 & 0.1 & 1.2 & 0.6 & 1.3 & 0.4 & 1.2 & 0.4 & 1.2 & - & 0.93 \\
\hline$\geq 2.0 \mathrm{mmol} / \mathrm{L}$ & $2(4.2)$ & - & $0(0.0)$ & - & $1(4.2)$ & - & $1(9.1)$ & - & $0(0.0)$ & - & $0(0.0)$ & - & - \\
\hline
\end{tabular}

BMI, body mass index; SES, socio-economic status; IRSAD, Index of Relative Socio-Economic Advantage and Disadvantage; ARFS, Australian Recommended Food Score; HLC, Healthy

Lifestyle Criteria; HDL-C, high density lipoprotein cholesterol; LDL-C, low density lipoprotein cholesterol. ${ }^{1} \mathrm{HDL}$ and LDL analysis conducted on 44 participants; TC and TG analysis conducted on 48 participants. 


\subsection{Linear Regression Analysis of Lifestyle Behaviours with CVD Risk Markers}

Linear regression of individual lifestyle behaviours and CVD risk markers are presented in Table 2. In the unadjusted models, physical activity was significantly and inversely associated with \%body fat $(\beta=-0.011 \%, p=0.005)$, waist circumference $(\beta=-0.013 \mathrm{~cm}, p=0.029)$ and diastolic blood pressure $(\beta=-0.010 \mathrm{mmHg}, p=0.031)$. A significant inverse association was also identified between alcohol intake and BMI $(\beta=-0.458, p=0.012)$, waist circumference $(\beta=-1.673 \mathrm{~cm}, p=0.003)$ and diastolic blood pressure $(\beta=-0.988 \mathrm{mmHg}, p=0.039)$. In the adjusted models, controlling for the other lifestyle behaviours, physical activity was no longer significantly associated with CVD risk markers. However, alcohol intake remained significantly and inversely associated with BMI $(\beta=-0.412, p=0.036)$ and waist circumference $(\beta=-1.389 \mathrm{~cm}, p=0.023)$.

Table 2. Linear regression analysis results of lifestyle behaviours with CVD risk markers in young (18-35 years) overweight and obese women (BMI $25-34.9 \mathrm{~kg} / \mathrm{m}^{2}$ ) entering a weight-loss trial.

\begin{tabular}{|c|c|c|c|c|c|c|}
\hline \multirow{2}{*}{ Variable } & \multicolumn{3}{|c|}{ Unadjusted Model $^{1}$} & \multicolumn{3}{|c|}{ Full-Adjusted Model ${ }^{2}$} \\
\hline & $\beta$-Coefficient ${ }^{3}$ & SE & $p$ & $\beta$-Coefficient & SE & $p$ \\
\hline \multicolumn{7}{|l|}{ BMI $\left(\mathrm{kg} / \mathrm{m}^{2}\right)$} \\
\hline ARFS & 0.014 & 0.051 & 0.780 & 0.087 & 0.054 & 0.113 \\
\hline Alcohol intake (standard drinks/day) & -0.458 & 0.174 & 0.012 * & -0.412 & 0.188 & $0.036^{*}$ \\
\hline Physical activity (min/week) & -0.003 & 0.002 & 0.073 & -0.004 & 0.002 & 0.124 \\
\hline Average sitting time (min/day) & 0.001 & 0.002 & 0.664 & -0.001 & 0.002 & 0.766 \\
\hline \multicolumn{7}{|l|}{ Body fat (\%) } \\
\hline ARFS & -0.108 & 0.103 & 0.303 & 0.035 & 0.111 & 0.757 \\
\hline Alcohol intake (standard drinks/day) & -0.735 & 0.373 & 0.056 & -0.410 & 0.391 & 0.301 \\
\hline Physical activity (min/week) & -0.011 & 0.004 & $0.005 *$ & -0.009 & 0.005 & 0.065 \\
\hline Average sitting time (min/day) & 0.006 & 0.004 & 0.143 & 0.003 & 0.004 & 0.523 \\
\hline \multicolumn{7}{|l|}{ Waist circumference $(\mathrm{cm})$} \\
\hline ARFS & -0.170 & 0.157 & 0.286 & 0.037 & 0.167 & 0.825 \\
\hline Alcohol intake (standard drinks/day) & -1.673 & 0.529 & 0.003 * & -1.389 & 0.586 & $0.023^{*}$ \\
\hline Physical activity (min/week) & -0.013 & 0.006 & $0.029 *$ & -0.009 & 0.007 & 0.210 \\
\hline Average sitting time (min/day) & 0.002 & 0.007 & 0.800 & -0.003 & 0.006 & 0.671 \\
\hline \multicolumn{7}{|l|}{ Systolic blood pressure (mmHg) } \\
\hline ARFS & -0.001 & 0.001 & 0.375 & -0.002 & 0.185 & 0.989 \\
\hline Alcohol intake (standard drinks /day) & -0.004 & 0.005 & 0.459 & -0.187 & 0.650 & 0.775 \\
\hline Physical activity (min/week) & -0.0001 & 0.0001 & 0.274 & -0.010 & 0.008 & 0.193 \\
\hline Average sitting time (min/day) & -0.0001 & 0.0001 & 0.165 & -0.013 & 0.007 & 0.074 \\
\hline \multicolumn{7}{|l|}{ Diastolic blood pressure $(\mathrm{mmHg})$} \\
\hline ARFS & -0.210 & 0.126 & 0.103 & -0.064 & 0.141 & 0.653 \\
\hline Alcohol intake (standard drinks/day) & -0.988 & 0.463 & $0.039 *$ & -0.653 & 0.496 & 0.197 \\
\hline Physical activity (min/week) & -0.010 & 0.005 & 0.031 * & -0.008 & 0.006 & 0.165 \\
\hline Average sitting time (min/day) & -0.002 & 0.005 & 0.758 & -0.005 & 0.005 & 0.342 \\
\hline \multicolumn{7}{|l|}{ Total cholesterol $(\mathrm{mmol} / \mathrm{L})^{4}$} \\
\hline ARFS & -0.017 & 0.016 & 0.288 & 0.001 & 0.001 & 0.267 \\
\hline Alcohol intake (standard drinks/day) & 0.046 & 0.058 & 0.432 & -0.002 & 0.003 & 0.389 \\
\hline Physical activity (min/week) & -0.00004 & 0.001 & 0.941 & 0.00001 & 0.00003 & 0.788 \\
\hline Average sitting time (min/day) & -0.0003 & 0.001 & 0.626 & 0.00002 & 0.00003 & 0.545 \\
\hline \multicolumn{7}{|l|}{ HDL cholesterol $(\mathrm{mmol} / \mathrm{L})^{4}$} \\
\hline ARFS & 0.002 & 0.008 & 0.851 & -0.007 & 0.010 & 0.483 \\
\hline Alcohol intake (standard drinks/day) & 0.045 & 0.034 & 0.199 & 0.038 & 0.039 & 0.334 \\
\hline Physical activity (min/week) & 0.0004 & 0.0003 & 0.250 & 0.0005 & 0.0004 & 0.275 \\
\hline Average sitting time (min/day) & 0.0001 & 0.0004 & 0.724 & 0.0004 & 0.0004 & 0.364 \\
\hline \multicolumn{7}{|l|}{ LDL cholesterol $(\mathrm{mmol} / \mathrm{L})^{4}$} \\
\hline ARFS & -0.006 & 0.005 & 0.175 & -0.015 & 0.016 & 0.338 \\
\hline Alcohol intake (standard drinks/day) & -0.006 & 0.020 & 0.781 & -0.009 & 0.062 & 0.889 \\
\hline Physical activity (min/week) & -0.0001 & 0.0002 & 0.714 & -0.00005 & 0.001 & 0.934 \\
\hline Average sitting time (min/day) & -0.0003 & 0.0002 & 0.199 & -0.001 & 0.001 & 0.194 \\
\hline \multicolumn{7}{|l|}{ Triglycerides $(\mathrm{mmol} / \mathrm{L})^{4}$} \\
\hline ARFS & 0.007 & 0.005 & 0.169 & 0.004 & 0.005 & 0.516 \\
\hline Alcohol intake (standard drinks/day) & 0.005 & 0.018 & 0.761 & -0.009 & 0.019 & 0.648 \\
\hline Physical activity (min/week) & 0.0003 & 0.0002 & 0.086 & 0.0003 & 0.0002 & 0.217 \\
\hline Average sitting time (min/day) & -0.0001 & 0.0002 & 0.737 & 0.00004 & 0.0002 & 0.865 \\
\hline
\end{tabular}

CVD, cardiovascular disease; BMI, body mass index; SE, standard error; ARFS; Australian Recommended Food

Score; HDL cholesterol, high density lipoprotein cholesterol; LDL cholesterol, low density lipoprotein cholesterol.

1 The unadjusted model includes the individual lifestyle behaviour and age, ethnicity and socio-economic status; ${ }^{2}$ The fully adjusted model includes all lifestyle behaviours (diet quality, alcohol intake, physical activity, sitting time) and age, ethnicity and socio-economic status; ${ }^{3} \beta$-Coefficient indicates the increase in the CVD risk marker per unit increase in the independent variable; ${ }^{4}$ HDL and LDL cholesterol analysis conducted on 44 participants; Total cholesterol and triglycerides analysis conducted on 48 participants; ${ }^{*}$ Indicates statistically significant result $(p<0.05)$. 


\subsection{Healthy Lifestyle Score}

All participants had a HLS of at least one, as all were non-smokers. The percentage of participants scoring 1, 2, 3, 4 and 5 was $10.2 \%, 49.0 \%, 24.5 \%, 14.3 \%$ and $2.0 \%$ respectively. Table 1 presents participants socio-demographic characteristics, lifestyle behaviours and CVD risk markers, by HLS (1-5). There were no significant differences in socio-demographic characteristics by HLS. Linear regression analyses exploring the association between HLS and each CVD risk marker found no significant associations (Table 3).

Table 3. Linear regression analysis results for CVD risk markers with Healthy Lifestyle Score in young (18-35 years) overweight and obese women (BMI $\left.25-34.9 \mathrm{~kg} / \mathrm{m}^{2}\right)$ entering a weight loss trial $\left(n=49^{1}\right)$.

\begin{tabular}{cccc}
\hline Variable & $\beta$-Coefficient ${ }^{2}$ & SE & $p$ \\
\hline BMI $\left(\mathrm{kg} / \mathrm{m}^{2}\right)$ & 0.419 & 0.464 & 0.372 \\
Body fat $(\%)$ & -0.542 & 0.965 & 0.577 \\
Waist circumference $(\mathrm{cm})$ & 0.695 & 1.468 & 0.639 \\
Systolic blood pressure $(\mathrm{mmHg})$ & 0.0001 & 0.0001 & 0.283 \\
Diastolic blood pressure $(\mathrm{mmHg})$ & -1.236 & 1.378 & 0.375 \\
Total cholesterol (mmol/L) & 0.027 & 0.147 & 0.856 \\
HDL cholesterol (mmol/L) & -0.047 & 0.079 & 0.550 \\
LDL cholesterol (mmol/L) & 0.009 & 0.045 & 0.851 \\
Triglycerides (mmol/L) & 0.019 & 0.053 & 0.729 \\
\hline
\end{tabular}

CVD, cardiovascular disease; BMI, body mass index; SE, standard error; HDL cholesterol, high density lipoprotein cholesterol; LDL cholesterol, low density lipoprotein cholesterol. ${ }^{1}$ HDL and LDL cholesterol analysis conducted on 44 participants; total cholesterol and triglycerides analysis conducted on 48 participants;

${ }^{2} \beta$-coefficient indicates the increase in the CVD risk marker per unit increase in the independent variable;

${ }^{3}$ Models adjusted for age, ethnicity and socio-economic status.

\section{Discussion}

In this cross-sectional analysis, most young (18-35 years) overweight and obese women reported multiple unhealthy diet, alcohol, physical activity and sitting time behaviours. The majority of participants had a Healthy Lifestyle Score of two or less, with only one participant with the maximum score of five. Many of the participants also had adverse CVD risk markers, particularly waist circumference and plasma total, HDL and LDL cholesterol. The key association identified between lifestyle behaviour and CVD risks was that insufficient physical activity was associated with increased body fat, waist circumference and diastolic blood pressure. These findings suggest that targeting physical activity should be a priority for health interventions for this population. Overall the study participants were similar compared to the broader population of young adult women in Australia with respect to percentage meeting physical activity guidelines [9]; however, more study participants exceeded alcohol guidelines [44] and fewer were smokers [45]. Diet quality was similar to participants of a comparable Australian study in overweight and obese young adults [46]. More of the current study participants had elevated CVD risk markers, including higher percentages with elevated waist circumference [47] and total cholesterol [42], and low HDL cholesterol [42] than young Australian adults.

The current study found a significantly lower \%body fat of $0.011 \%(p=0.005)$, smaller waist circumference of $0.013 \mathrm{~cm}(p=0.029)$ and lower diastolic blood pressure of $0.010 \mathrm{mmHg}(p=0.031)$ for every additional minute per week of moderate or vigorous physical activity. Despite these results only being significant in the unadjusted models, this association is consistent with previous cross-sectional and longitudinal studies demonstrating significant associations with higher physical activity and lower waist circumference and \%body fat [48-52], including studies in young adult women [49,52]. Healy et al. also found higher physical activity to be associated with lower total: HDL cholesterol ratio and triglycerides, and increased HDL cholesterol [50]. These studies in middle-aged adults [48,50,51] also indicated a trend of lower blood pressure with increasing physical activity; however, statistical 
significance of the associations for systolic and diastolic blood pressure varied between the findings. The current study supports these findings, and additionally suggests that physical activity levels are impacting CVD risk markers in populations of younger overweight and obese women. Therefore, the findings provide further support for the need for physical activity interventions for young overweight and obese women.

Overall, the current study found no associations between diet quality or sitting time and CVD risk markers, and unexpectedly found an association between a higher alcohol intake and lower BMI, waist circumference and diastolic blood pressure. Conversely previous longitudinal studies of young and middle-aged women have reported associations between higher alcohol intake and increased hypertension and hypercholesterolaemia risk [22,53], as well as higher diet quality and lower hypertension risk [22] and weight gain [54]. Additionally previous cross-sectional studies have reported associations between higher sitting time and increased risk of higher waist circumference in young adult women [49] and overweight or obesity in young and middle-aged men and women [55,56]. The cross-sectional nature and inclusion criteria of the current study could partly explain the difference in findings. For example, the exclusion of healthy weight participants could have limited the comparative ability of the analyses. It is also possible that participants with higher BMI were trying to reduce alcohol intake, particularly as a means to achieve weight loss. Despite the current study findings regarding alcohol intake, the participants' alcohol consumption is high and exceeds that of the wider young adult female population. As such health messages to reduce alcohol intake would be a pertinent addition to lifestyle interventions in this group.

The association between a higher number of high risk lifestyle behaviours and greater CVD risk has been demonstrated in several observational studies [17-22,25], including the cross-sectional analysis of young Australian adults by Gall et al. [25]. The current study found no associations between Healthy Lifestyle Score and CVD risk markers. With the exception of Gall et al. the other studies explored lifestyle behaviours in young adulthood and their combined impact on CVD risk markers 10 to 22 years later, hence the cross-sectional nature of the current analysis could account for the lack of significant findings. The HLS is a non-validated score, which was developed for the purposes of this analysis. There is a need for validated composite measures in this field that consider the relationship with health outcomes in large population samples. Other limitations of the study may also have impacted the results. The small sample size and the RCT inclusion/exclusion criteria may have limited the range of lifestyle behaviours and CVD risk markers assessed. Also, participants were enrolling in a weight loss study; they were not a random sample from the general population, which limits generalisability. The strict inclusion criteria may also have limited recruitment and the sample size as, despite formative research with the target group, recruitment and retention were lower than anticipated [26,57]. Additionally as a consequence of sample size, the study was not powered for all analyses. Other limitations should also be acknowledged when interpreting the results. As there are no current population-based recommendations for appropriate ARFS or sitting time the criteria for these were based on the distribution of the study participants' data. Smokers were not represented, which may partially reflect the over representation of young women who were engaged in tertiary education studies [58]. Participants' ability to recall dietary and alcohol intakes over a six-month period is potentially limiting. However, previous studies have used this time frame or longer [25], and the Australian Eating Survey has been validated over this time frame [27]. Finally, no data were obtained regarding participants' family history of CVD or use of medications to treat elevated CVD risk factors. This information could have identified participants already at an increased risk of CVD. Future studies should obtain this information and include it in analyses as a confounder.

The strengths of the current study include the use of objective measures for all CVD risk markers (BMI, \%body fat, waist circumference, blood pressure, plasma total, HDL and LDL cholesterol and triglycerides). Although some research has questioned the validity of bioelectrical impedance as a measure of body fat [59], the instrument used in the current study has comparative validity to dual-energy X-ray absorptiometry measures, and tends to underestimate rather than overestimate 
\%body fat [60]. Secondly, the use of the AES FFQ, ARFS, Domain-Specific Sitting Time Questionnaire and Godin Leisure Time Exercise Questionnaire, all validated in middle and/or older-aged Australian women $[27,28,32,61]$, reduces the potential bias from participants' self-reporting these behaviours. In addition, the current study sample was representative of young adult women in Australia in terms of Aboriginality [62] and income [63].

\section{Conclusions}

The current study reinforces that unhealthy lifestyle behaviours and cardiovascular disease (CVD) risk markers are prevalent in young overweight and obese women. The major finding is that physical activity levels are directly associated with increased CVD risk markers. In addition, these young women are consuming high amounts of alcohol. This suggests that lifestyle interventions, especially those including physical activity, targeting young overweight and obese women are warranted and have the potential to improve cardiovascular risk factors and heart health across the adult life course.

Acknowledgments: This study was funded by a University of Newcastle New Staff Grant. Melinda Hutchesson is supported by a National Heart Foundation Postdoctoral Research Fellowship, and C Collins is supported by a NHMRC Senior Research Fellowship.

Author Contributions: Melinda J. Hutchesson, Robin Callister and Clare E. Collins were responsible for the study design. Megan C. Whatnall conducted the statistical analysis with the assistance of Melinda J. Hutchesson. Megan C. Whatnall and Melinda J. Hutchesson drafted the initial manuscript. All authors contributed to interpreting the results, reviewing, editing and approving the final manuscript.

Conflicts of Interest: The authors declare no conflict of interest.

\section{References}

1. World Health Organization. Women's Health. Available online: http://www.who.int/mediacentre/ factsheets/fs334/en/ (accessed on 1 March 2016).

2. World Health Organization. Global Status Report on Noncommunicable Diseases 2014; World Health Organization: Geneva, Switzerland, 2014.

3. World Health Organization. Cardiovascular Diseases-Key Facts. Available online: http://www.who.int/ mediacentre/factsheets/fs317/en/ (accessed on 10 June 2016).

4. Katzmarzyk, P.T.; Church, T.S.; Craig, C.L.; Bouchard, C. Sitting time and mortality from all causes, cardiovascular disease, and cancer. Med. Sci. Sports Exerc. 2009, 41, 998-1005. [CrossRef] [PubMed]

5. Patel, A.V.; Bernstein, L.; Deka, A.; Feigelson, H.S.; Campbell, P.T.; Gapstur, S.M.; Colditz, G.A.; Thun, M.J. Leisure time spent sitting in relation to total mortality in a prospective cohort of us adults. Am. J. Epidemiol. 2010, 172, 419-429. [CrossRef] [PubMed]

6. Australian Institute of Health and Welfare. Women and Heart Disease: Cardiovascular Profile of Women in Australia. Available online: http:/ /www.aihw.gov.au/WorkArea/DownloadAsset.aspx?id=6442455107 (accessed on 1 February 2016).

7. The Heart Foundation. Heart Disease: Scope and Impact. Available online: http://www.theheartfoundation. org/heart-disease-facts/heart-disease-statistics / (accessed on 1 March 2016).

8. World Heart Federation. Cardiovascular Disease-Risk Factors. Available online: http://www.world-heartfederation.org/fileadmin/user_upload/documents/Fact_sheets/2012/PressBackgrounderApril2012RiskFactors. pdf (accessed on 1 March 2016).

9. Australian Bureau of Statistics. 4364.0.55.004-Australian Health Survey: Physical Activity, 2011-12. Available online: http:/ /www.abs.gov.au/ausstats/abs@.nsf/Lookup/D4495467B7F7EB01CA257BAC0015F593 (accessed on 20 May 2016).

10. Australian Bureau of Statistics. 4338.0-Profiles of Health, Australia, 2011-13. Daily Intake of Fruit and Vegetables. Available online: http://www.abs.gov.au/ausstats/abs@.nsf/Lookup/by\%20Subject/4338. 0 2011-13 Main\%20Features Daily\%20intake\%20of\%20fruit\%20and\%20vegetables 10009 (accessed on 1 October 2015).

11. World Health Organization. Global Health Observatory Data-Prevalence of Tobacco Use. Available online: http:/ /www.who.int/gho/tobacco/use/en/ (accessed on 1 June 2016). 
12. Centers for Disease Control and Prevention. Current Cigarette Smoking among Adults in the United States. Available online: http://www.cdc.gov/tobacco/data_statistics/fact_sheets/adult_data/cig_smoking/ index.htm\#national (accessed on 1 March 2016).

13. Australian Bureau of Statistics. 4125.0—Gender Indicators, Australia, Jan 2013-Smoking. Available online: http:/ /www.abs.gov.au/ausstats/abs@.nsf/Lookup/4125.0main+features3320Jan\%202013 (accessed on 1 March 2016).

14. National Institute on Alcohol Abuse and Alcoholism. Alcohol-A Women's Health Issue. Available online: http://pubs.niaaa.nih.gov/publications/brochurewomen/women.htm (accessed on 30 April 2016).

15. Australian Bureau of Statistics. 4364.0.55.001-Australian Health Survey: First Results, 2011-2012-Alcohol Consumption. Available online: http://www.abs.gov.au/ausstats/abs@.nsf/Latestproducts/ D522399EBE2DAB46CA257AA30014BE96?opendocument (accessed on 1 June 2016).

16. Brown, W.J.; Williams, L.; Ford, J.H.; Ball, K.; Dobson, A.J. Identifying the energy gap: Magnitude and determinants of 5-year weight gain in midage women. Obes. Res. 2005, 13, 1431-1441. [CrossRef] [PubMed]

17. Larsson, S.C.; Akesson, A.; Wolk, A. Healthy diet and lifestyle and risk of stroke in a prospective cohort of women. Neurology 2014, 83, 1699-1704. [CrossRef] [PubMed]

18. Odegaard, A.O.; Koh, W.P.; Gross, M.D.; Yuan, J.M.; Pereira, M.A. Combined lifestyle factors and cardiovascular disease mortality in chinese men and women: The singapore chinese health study. Circulation 2011, 124, 2847-2854. [CrossRef] [PubMed]

19. Stamler, J.; Stamler, R.; Neaton, J.D.; Wentworth, D.; Daviglus, M.L.; Garside, D.; Dyer, A.R.; Liu, K.; Greenland, P. Low risk-factor profile and long-term cardiovascular and noncardiovascular mortality and life expectancy: Findings for 5 large cohorts of young adult and middle-aged men and women. JAMA 1999, 282, 2012-2018. [CrossRef] [PubMed]

20. Yang, Q.; Cogswell, M.E.; Flanders, W.D.; Hong, Y.; Zhang, Z.; Loustalot, F.; Gillespie, C.; Merritt, R.; Hu, F.B. Trends in cardiovascular health metrics and associations with all-cause and cvd mortality among us adults. JAMA 2012, 307, 1273-1283. [CrossRef] [PubMed]

21. Liu, K.; Daviglus, M.L.; Loria, C.M.; Colangelo, L.A.; Spring, B.; Moller, A.C.; Lloyd-Jones, D.M. Healthy lifestyle through young adulthood and the presence of low cardiovascular disease risk profile in middle age: The coronary artery risk development in (young) adults (cardia) study. Circulation 2012, 125, 996-1004. [CrossRef] [PubMed]

22. Chomistek, A.K.; Chiuve, S.E.; Eliassen, A.H.; Mukamal, K.J.; Willett, W.C.; Rimm, E.B. Healthy lifestyle in the primordial prevention of cardiovascular disease among young women. J. Am. Coll. Cardiol. 2015, 65, 43-51. [CrossRef] [PubMed]

23. O'Flaherty, M.; Allender, S.; Taylor, R.; Stevenson, C.; Peeters, A.; Capewell, S. The decline in coronary heart disease mortality is slowing in young adults (australia 1976-2006): A time trend analysis. Int. J. Cardiol. 2012, 158, 193-198. [CrossRef] [PubMed]

24. Australian Bureau of Statistics. 4364.0.55.004-Australian Health Survey: Physical Activity, 2011-2012. Pedometer Steps. Available online: http://www.abs.gov.au/ausstats/abs@.nsf/Lookup/4364.0.55. 004Chapter5002011-12 (accessed on 2 October 2015).

25. Gall, S.L.; Jamrozik, K.; Blizzard, L.; Dwyer, T.; Venn, A. Healthy lifestyles and cardiovascular risk profiles in young Australian adults: The childhood determinants of adult health study. Eur. J. Cardiovasc. Prev. Rehabil. 2009, 16, 684-689. [CrossRef] [PubMed]

26. Leonard, A.; Hutchesson, M.; Patterson, A.; Chalmers, K.; Collins, C. Recruitment and retention of young women into nutrition research studies: Practical considerations. Trials 2014. [CrossRef] [PubMed]

27. Collins, C.E.; Boggess, M.M.; Watson, J.F.; Guest, M.; Duncanson, K.; Pezdirc, K.; Rollo, M.; Hutchesson, M.J.; Burrows, T.L. Reproducibility and comparative validity of a food frequency questionnaire for australian adults. Clin. Nutr. 2014, 33, 906-914. [CrossRef] [PubMed]

28. Collins, C.E.; Burrows, T.L.; Rollo, M.E.; Boggess, M.M.; Watson, J.F.; Guest, M.; Duncanson, K.; Pezdirc, K.; Hutchesson, M.J. The comparative validity and reproducibility of a diet quality index for adults: The australian recommended food score. Nutrients 2015, 7, 785-798. [CrossRef] [PubMed]

29. National Health and Medical Research Council. Australian Dietary Guidelines; National Health and Medical Research Council: Canberra, Australia, 2013. 
30. Centre for Epidemiology and Evidence. Nsw Population Health Survey 2013 Questionnaire. Available online: http:/ / www.health.nsw.gov.au/surveys/adult/Documents/Questionnaire-2013-WEB.pdf (accessed on 2 May 2016).

31. Godin, G.; Shephard, R. Godin leisure-time exercise questionnaire. Med. Sci. Sports Exerc. 1997, $29,36-38$.

32. Marshall, A.L.; Miller, Y.D.; Burton, N.W.; Brown, W.J. Measuring total and domain-specific sitting: A study of reliability and validity. Med. Sci. Sports Exerc. 2010, 42, 1094-1102. [CrossRef] [PubMed]

33. National Health and Medical Research Council. Australian Guidelines to Reduce Health Risks from Drinking Alcohol; National Health and Medical Research Council: Canberra, Australia, 2009.

34. National Heart Foundation of Australia. Walking-Making Every Step Count. Available online: http: //www.heartfoundation.org.au/SiteCollectionDocuments/HFW-Make-Every-Step-Count.pdf (accessed on 1 September 2015).

35. Australian Government, Department of Health. Australia's Physical Activity and Sedentary Behaviour Guidelines. Available online: http://www.health.gov.au/internet/main/publishing.nsf/content/healthpubhlth-strateg-phys-act-guidelines (accessed on 2 May 2016).

36. World Health Organization. Obesity: Preventing and Managing the Global Epidemic. Report of a Who Consultation; World Health Organization: Geneva, Switzerland, 2000.

37. Panz, V.R.; Raal, F.J.; Paiker, J.; Immelman, R.; Miles, H. Performance of the cardiochek pa and cholestech ldx point-of-care analysers compared to clinical diagnostic laboratory methods for the measurement of lipids. Cardiovasc. J. S. Afr. 2005, 16, 112-117. [PubMed]

38. Ferreira, C.E.; Franca, C.N.; Correr, C.J.; Zucker, M.L.; Andriolo, A.; Scartezini, M. Clinical correlation between a point-of-care testing system and laboratory automation for lipid profile. Clin. Chim. Acta 2015, 446, 263-266. [CrossRef] [PubMed]

39. Australian Bureau of Statistics. Technical Paper: Socio-Economic Indexes for Areas (Seifa); Australian Bureau of Statistics: Canberra, Australia, 2013.

40. World Health Organization. Waist Circumference and Waist-Hip Ratio. Report of a Who Expert Consultation; World Health Organization: Geneva, Switzerland, 2008.

41. National Heart Foundation of Australia. Guide to Management of hypertension 2008. Updated December 2010; National Heart Foundation of Australia: Canberra, Australia, 2010.

42. Australian Bureau of Statistics. 4364.0.55.005-Australian Health Survey: Biomedical Results for Chronic Diseases, 2011-12-Cholesterol. Available online: http://www.abs.gov.au/ausstats/abs@.nsf/lookup/ 4812278BC4B8FE1ECA257BBB001217A4?opendocument (accessed on 1 September 2015).

43. Australian Bureau of Statistics. 4364.0.55.005-Australian Health Survey: Biomedical results for Chronic Diseases, 2011-12-Triglycerides. Available online: http:/ /www.abs.gov.au/ausstats/abs@.nsf/ Latestproducts/840CB1F3C029B632CA257BBB00121823?opendocument (accessed on 30 September 2015).

44. Australian Bureau of Statistics. 4338.0-Profiles of Health, Australia, 2011-13-Alcohol Consumption. Available online: http://www.abs.gov.au/ausstats/abs@.nsf/Lookup/4338.0main+features62011-13 (accessed on 30 September 2015).

45. Australian Bureau of Statistics. 4125.0 Gender Indicators, Australia, January 2013. Available online: http: //www.abs.gov.au/AUSSTATS/abs@.nsf/DetailsPage/4125.0Jan\%202013?OpenDocument (accessed on 1 September 2015).

46. Nour, M.M.; McGeechan, K.; Wong, A.T.; Partridge, S.R.; Balestracci, K.; Roy, R.; Hebden, L.; Allman-Farinelli, M. Diet quality of young adults enrolling in txt2bfit, a mobile phone-based healthy lifestyle intervention. JMIR Res. Protoc. 2015, 4, e60. [CrossRef] [PubMed]

47. Australian Bureau of Statistics. 4364.0.55.001-Australian health Survey: First Results, 2011-12-Waist Circumference. Available online: http://www.abs.gov.au/ausstats/abs@.nsf/Latestproducts/ 6A2304311987758FCA257AA30014C0C6?opendocument (accessed on 1 September 2015).

48. Chan, C.B.; Spangler, E.; Valcour, J.; Tudor-Locke, C. Cross-sectional relationship of pedometer-determined ambulatory activity to indicators of health. Obes. Res. 2003, 11, 1563-1570. [CrossRef] [PubMed]

49. Cleland, V.; Schmidt, M.; Salmon, J.; Dywer, T.; Venn, A. Combined associations of sitting time and physical activity with obesity in young adults. J. Phys. Act. Health 2014, 11, 136-144. [CrossRef] [PubMed]

50. Healy, G.N.; Winkler, E.A.H.; Owen, N.; Anuradha, S.; Dunstan, D.W. Replacing sitting time with standing or stepping: Associations with cardio-metabolic risk biomarkers. Eur. Heart J. 2015, 36, 2643-2649. [CrossRef] [PubMed] 
51. Pillay, J.D.; Kolbe-Alexander, T.L.; van Mechelen, W.; Lambert, E.V. Steps that count: The association between the number and intensity of steps accumulated and fitness and health measures. J. Phys. Act. Health 2014, 11, 10-17. [CrossRef] [PubMed]

52. Morinaka, T.; Limtrakul, P.-N.; Makonkawkeyoon, L.; Sone, Y. Comparison of variations between percentage of body fat, body mass index and daily physical activity among young japanese and thai female students. J. Physiol. Anthropol. 2012, 31, 1-4. [CrossRef] [PubMed]

53. Sesso, H.D.; Cook, N.R.; Buring, J.E.; Manson, J.E.; Gaziano, J.M. Alcohol consumption and the risk of hypertension in women and men. Hypertension 2008, 51, 1080-1087. [CrossRef] [PubMed]

54. Aljadani, H.M.; Patterson, A.; Sibbritt, D.; Hutchesson, M.J.; Jensen, M.E.; Collins, C.E. Diet quality, measured by fruit and vegetable intake, predicts weight change in young women. J. Obes. 2013. [CrossRef] [PubMed]

55. Chau, J.Y.; van der Ploeg, H.P.; Merom, D.; Chey, T.; Bauman, A.E. Cross-sectional associations between occupational and leisure-time sitting, physical activity and obesity in working adults. Prev. Med. 2012, 54, 195-200. [CrossRef] [PubMed]

56. MacFarlane, A.M.; Abbott, G.R.; Crawford, D.A.; Ball, K. Sociodemographic and behavioural correlates of weight status among women with children living in socioeconomically disadvantaged neighbourhoods. Int. J. Obes. 2009, 33, 1289-1298. [CrossRef] [PubMed]

57. Hutchesson, M.J.; Morgan, P.J.; Callister, R.; Pranata, I.; Skinner, G.; Collins, C.E. Be positive be healthe: Development and implementation of a targeted e-health weight loss program for young women. Telemed. J. E. Health 2016, 22, 519-528. [CrossRef] [PubMed]

58. Australian Bureau of Statistics. 4102.0-Australian Social Trends, April 2013-Young Adults: Then and Now. Available online: http:/ / www.abs.gov.au/AUSSTATS/abs@.nsf/Lookup/4102.0Main+Features40April+ 2013 (accessed on 1 September 2015).

59. Franco-Villoria, M.; Wright, C.M.; McColl, J.H.; Sherriff, A.; Pearce, M.S. Gateshead Millennium Study Core Team. Assessment of adult body composition using bioelectrical impedance: Comparison of researcher calculated to machine outputted values. BMJ Open 2016, 6, e008922. [CrossRef] [PubMed]

60. Volgyi, E.; Tylavsky, F.A.; Lyytikainen, A.; Suominen, H.; Alen, M.; Cheng, S. Assessing body composition with dxa and bioimpedance: Effects of obesity, physical activity, and age. Obesity 2008, 16, 700-705. [CrossRef]

61. Godin, G.; Shephard, R.J. A simple method to assess exercise behavior in the community. Can. J. Appl. Sport Sci. 1985, 10, 141-146. [PubMed]

62. Australian Bureau of Statistics. 3238.0.55.001-Estimates of Aboriginal and Torres Strait Islander Australians, June 2011. Available online: http:/ /www.abs.gov.au/ausstats/abs@.nsf/mf/3238.0.55.001 (accessed on 1 September 2015).

63. Australian Bureau of Statistics. 6310.0-Employee Earnings, Benefits and Trade Union Membership, Australia. Available online: http://www.ausstats.abs.gov.au/Ausstats/subscriber.nsf/0/ 0E2A2378A582DFFECA257B6D00129B37/ \protect $\backslash T 1 \backslash$ textdollarFile/63100_august\%202012.pdf (accessed on 1 September 2015).

(C) 2016 by the authors; licensee MDPI, Basel, Switzerland. This article is an open access article distributed under the terms and conditions of the Creative Commons Attribution (CC-BY) license (http://creativecommons.org/licenses/by/4.0/). 